\title{
RELACIÓN QUE SE ESTABLECE ENTRE LA COMUNIDAD DE VIÑALES Y EL PARQUE NACIONAL VIÑALES
}

\author{
por \\ MARTÍN HERNÁNDEZ, J. VALDIVIA, M.A. Y DOMINGUEZ, M.
}

\section{RESUMEN}

Ante la presencia de población dentro o cercana a las Areas Protegidas, se hace necesario la asimilación, del proceso de protección de la naturaleza en estas comunidades, como principio básico para lograr un uso sustentable de los recursos naturales. Por lo que este trabajo tuvo como principal objetivo determinar las relaciones que se establecen entre la comunidad de Viñales y el Parque Nacional Viñales. Conociendo estas relaciones y las opiniones de los habitantes de la comunidad acerca del Parque se creó un plan de acción para educar y concientizar ambientalmente a sus habitantes, con el fin de lograr su protección.

PALABRAS CLAVES: áreas protegidas, comunidades, parque nacional, educación ambiental.

\section{ABSTRACT}

Faced with the presence of population within or near the Protected Areas, the assimilation of the nature protection process becomes necessary in these communities, as a basic principle to achieve a sustainable use of the natural resources. That is why the main objective of this work was to determine the relations that are established between the community of Viñales and the Viñales National Park. After discovering these relations and the opinions of the inhabitants of the community about the park, a plan of action was created to educate the inhabitants and make them environmentally aware, in order to achieve its protection.

KEY WORDS: Protected areas; communities; natural park; environmental education.

\section{INTRODUCCION}

En el ámbito mundial se va tomando conciencia de la necesidad de proteger la Naturaleza, ya que es vital para encontrar el camino al desarrollo sustentable. Una de las formas es la 
implantación de Areas Protegidas. Internacionalmente desde que se comenzaron a declarar a éstas, como Parques Nacionales para la protección de sus recursos, siempre ha sido un problema la presión ejercida por el hombre para explotar los que están a su alcance.

El hombre y las áreas protegidas no son independientes, la población humana forma parte de un todo integral de casi todos los ecosistemas vivos. Por lo que cualquier plan que quiera asegurar el crecimiento y la supervivencia de los ecosistemas debe tener en cuenta e incluir a la población que en ellos vive, sino quiere fracasar.

Muestra de esta presencia humana es que casi el $86 \%$ de los Parques Nacionales de América del Sur tienen que enfrentar el problema de grupos poblacionales permanientes o temporales asentados dentro de sus límites(Amend, S. y T. 1992). Por lo que las Areas Protegidas no pueden coexistir a largo plazo con comunidades que le son hostiles.

Cuba no ha quedado al margen de este acontecimiento, llevando a cabo un amplio proceso de conservación que comenzó a tener fuerza después de la creación del Ministerio de Ciencia Tecnología y Medio Ambiente (CITMA). Actualmente ya se han comenzado a dar pasos para formar las bases de una cultura ambiental en el país con la elaboración de la nueva Ley Marco sobre Medio Ambiente, Ley № 81 aprobada el 11 de julio de 1997 por la Asamblea Nacional del Poder Popular y a partir de ésta se están elaborando y aprobando decretos - leyes, que ayudan a la reformulación de la política ambiental en el país, la cual ha dado por resultado el Sistema Nacional de Areas Protegidas, donde esta incluido el Parque Nacional Viñales, en el cual sé esta realizando actualmente el estudio, de compatibilización y legalización para su implementación como tal.

\section{DESARROLLO}

El Parque Nacional «Viñales» se encuentra ubicado en la mitad occidental de la Cordillera de Guaniguanico, provincia Pinar del Río, encerrando dentro de sus límites una porción de las alturas y montañas bajas de la Sierra de los Organos, que limita al norte con la base de la sierra cársica, al sur con las pizarras de la formación San Cayetano y siguiendo al este y oeste los límites transicionales en los contactos entre pizarras y calizas. Cuenta con una extensión de $146,25 \mathrm{~km} 2$, donde se localizan todos los posibles grupos de paisajes naturales cársicos, o sea, mogotes aislados, sierras calcáreas y carso ruiniforme. Es un área de estudio clásico del 
desarrollo del carso cónico y de torres en los trópicos.

Puede citarse, por ejemplo, que dentro del mismo están dos de los tres sistemas cavernarios mayores de Cuba, sitios muy importantes de asentamientos aborígenes y de cimarronería del Occidente Cubano, y concentrados muchos de los valores biológicos tanto de flora como de fauna en estos tipos de paisajes, con gran número de endemismos.

Aunque existía población en el área donde hoy se encuentra enclavada la población de Viñales, no es hasta el año 1879 donde se autoriza la creación dẹ término municipal y la población comienza a crecer, lo que dura durante todo el resto del siglo XIX, llegando incluso a tener un ferrocarril. La población era fundamentalmente de origen hispano, lo cual hoy día se comprueba entre los habitantes actuales. El pueblo tiene una interesantísima arquitectura basada en casas de bajos techos, gruesas columnas y tejas muy peculiares, lo que ha llegado hasta nuestros días, como consecuencia de la recesión económica de la II década del siglo XX y que demoró hasta 1959. El pueblo no creció y no tuvo transformaciones, lo que posibilitó la conservación y el mantenimiento del estilo colonial en las construcciones de Vinales.

Dentro del área que ocupa la propuesta del Parque Nacional Viñales, se encuentran extensas áreas naturales de difícil acceso, donde conviven con la naturaleza en los territorios de más fácil asimilación agrícola un considerable número de habitantes, agrupados en dos núcleos urbanos, ocho asentamientos humanos rurales y abundante población dispersa, constituida fundamentalmente por campesinos independientes, donde no siempre se establece una relación armónica en el proceso de desarrollo, nivel de bienestar humano y utilización de los recursos naturales.

Las principales actividades económicas que explotan los recursos naturales de la región del municipio de Viñales en la actualidad son fundamentalmente actividades primarias como la agricultura, destacándose el cultivo del tabaco, la ganadería y la explotación forestal, a parte de otras que se desarrollan en la Empresa de Industrias Locales como la artesanía y otros productos, y por ultimo podemos mencionar el turismo, que en la actualidad ocupa un lugar de vital importancia para la economía del municipio y sobre todo en el futuro.

Las prácticas de explotación agrícola conservan hoy día la tradicionalidad, las cuales se han mantenido de generación en generación en el campo del desarrollo, conservación y uso sostenible de los recursos naturales.

En este contexto, es factible la búsqueda de alternativas de manejo sostenible en colaboración con las comuni- 
dades en el área del parque. Se trata de poner en juego todos aquellos recursos existentes en el medio rural, tanto materiales como culturales, para lograr el desarrollo sustentable. Hay que buscar la integración del fin económico con el objetivo más amplio de dinamización social y cultural de las comunidades.

Este trabajo se centro en el núcleo urbano de Viñales que es el más importante por ser la cabecera municipal, con una población, mayor de 6000 habitantes. Por ello, la investigación realizada tuvo como objetivos determinar las relaciones que se establecen entre la población residente en el asentamiento urbano de Viñales con el Parque Nacional y establecer un plan de acción para lograr la educación y concientización ambiental de dicha población

Durante la búsqueda y selección del método a utilizar en la investigación se consideraron dos aspectos: primero qué información se necesitaba obtener sobre la vida y las opiniones de la comunidad respecto al Parque Nacional y segundo cómo podría obtenerse dicha información, concluyéndose que la estaba en la aplicación de una encuesta semiabierta y de carácter anónimo.

La encuesta recoge la estructura sexo - etárea y nivel escolar de cada uno de los encuestados, y considerando el poder de decisión, la ocupación y qué relación o dependencia tienen de la naturaleza, y los beneficios que obtiene cada individuo de ésta, se determino el nivel de relación de cada encuestado con lo que será el Parque Nacional. También se persigue saber:

- ¿Qué conoce la comunidad de su entorno?

- ¿Qué provecho sacan de él?

- ¿Qué acción ejercería la población ante la instauración del Parque?

- ¿Qué opiniones tiene la comunidad sobre lo que traería consigo el Parque Nacional Viñales para su vida cotidiana?.

Problemas ambientales que la comunidad conoce y su posible solución:

- ¿Qué relación debe establecerse entre la comunidad y el Parque?

- ¿De qué forma cree la comunidad que debe recibir la información sobre el funcionamiento del Parqùe?

El análisis de los resultados obtenidos al procesar la información dada por la comunidad de Vinales posibilito determinar que el $76 \%$ de la población de Vinales constituida en un $90,7 \%$ por hombres entre 15 y 55 años son los que mayormente implicados se ven en el proceso de protección y conservación de la naturaleza, al ser los que más inte ractúan con su entorno, obteniendo ur mayor provecho del mismo. 
Para la población de Viñales los valores naturales más importantes son por orden de importancia: los valles, los mogotes, el paisaje, las cuevas, los cultivos y el clima. Como se puede apreciar los tres principales elementos naturales que las personas entrevistadas seleccionaron son aquellos que más conocen, , con los que están más tiempo en contacto, que es donde se desenvuelven sus vidas.

El $80 \%$ de los viñaleros consideran que conocen bien o algo las características de su entorno, aunque en ocasiones estos conocimientos se quedan en la descripción externa, aunque hay personas con una rica experiencia y conocimientos de la región. El 26\% de la población encuestada afirman que los conocimientos que tienen sobre la naturaleza de Viñales lo obtuvieron en la escuela, pero que ésta no es suficiente, más bien superficial.

Mediante la encuesta aplicada se pudieron determinar los beneficios que la población explota de su entorno. Algunos de estos beneficios apenas hacen daño a los valores naturales. Ejemplo de ellos son la recreación y satisfacción espiritual mediante la contemplación del paisaje que ocupa la preferencia de la inmensa mayoría de los viñaleros, lo saludable del clima y el medio ambiente. La tranquilidad del lugar unido al canto de las aves hacen del Valle de Viñales un lugar acogedor para sus habitantes.

Por otra parte, la existencia de necesidades materiales, propiciadas por la situación existente y la poca cultura ambiental, hacen que los daños que la población pueda ocasionarle a la naturaleza sean mayores, por ejemplo el incremento de cazadores furtivos

Otro de los de recursos de gran importancia para la población del pueblo de Viñales son sus suelos, base para la alimentación de sus habitantes por su alta fertilidad y sus recursos forestales, que además de ser explotados por el Estado, son utilizados para la construcción de viviendas por un $34 \%$ de su creciente población y un $26 \%$ admite haber suplido sus necesidades de combustible con la utilización de leña y carbón.

La entrada de turismo en la región por sus bellezas naturales beneficia al país y a la población de Viñales al crear empleos en el territorio, habiendo un $14 \%$ de habitantes en la región que obtienen beneficios de forma directa. Por lo que esta actividad ha traído consigo, un aumento en la extracción de materias primas para la artesanía con el fin de ofertarlas como suovenir, así como la comercialización de aves y caracoles de las especies de Vianas y Ligus, causando grandes daños a la fauna autóctona. 
Hasta el momento se han analizado los elementos naturales más importantes para los viñaleros y alguno de los beneficios que obtienen de ellos, por lo que es necesario conocer hasta que punto están dispuestos a conservarlos y preservarlos para el futuro, ante la declaración de Viñales como Parque Nacional. Para lograr esta percepción y criterios de cómo se beneficiarían o perjudicarían los habitantes de Viñales con el Parque se incluyó en la encuesta una pregunta que recoge esta información.

Los resultados de la misma arrojaron que el $97 \%$ de la población de la comunidad apoyan la declaración de Parque Nacional, aunque desconocen este concepto, además de qué significaría o qué importancia tiene su declaración para Viñales, es decir cómo influiría en su desarrollo. No obstante, un $68 \%$ de esta gran mayoría que lo apoyan están de acuerdo en trabajar por la conservación de la naturaleza, aunque muchos de ellos desconocen cómo proteger, conservar y mejorar el medio ambiente. Por lo que sólo argumentan que estarían dispuestos a cuidar y proteger a la naturaleza, velando por el restablecimiento de la flora y la fauna, evitando la tala de los bosques y la caza de especies en vías de extinción.

Muchas personas que trabajan en educación también plantearon su disposición de ayudar en la educación ambiental de los niños, estudiantes y adultos en general. Así como también en la protección del patrimonio colonial y cultural del pueblo.

Con el objetivo de conocer qué criterio tenía la población acerca de lo qué traería consigo el Parque Nacional Viñales, en el cuestionario aplicado, se les dio una serie de opciones que permitirían conocer cómo piensan las personas que viven dentro de la comunidad, de su futuro como habitantes dentro de un Area Protegida de grandes valores naturales a proteger.

Las personas siempre tendieron a marcar los beneficios que consideraban que de forma directa o indirecta mejorarían su situación, aunque muchos plantearon que algunas regulaciones $y$ limitantes necesarias a tener en cuenta, siempre serían para el beneficio del hombre.

El aumento del turismo de forma positiva para la población tuvo una aceptación del $80 \%$ aunque algunas personas plantearon que éste debía ser un turismo ecológico, otras que no estaban en contra de este aumento argu mentaban que no veían el beneficic hasta ahora y están los que quieren e aumento del turismo con fines lucrati vos acentuando tendencias negativas También hubo opiniones de que e turismo podría ser negativo, por lo que un $64 \%$ de la población planteó la necesidad de un aumento de opcione: recreativas. 
La posibilidad de aprender métodos de utilizar la naturaleza sin destruirla tuvo, junto con la posibilidad de elevar el nivel cultural de la población un $75 \%$ de aceptación, lo que demuestra la disposición de la comunidad de conservar la naturaleza sin dejar de utilizarla pero de una forma racional. Esto, unido a una cultura ambiental de sus habitantes, son los elementos necesarios para que el Parque fusione y en conjunto con la población de Viñales lograr un desarrollo sustentable. Lo que traería consigo, según un $67 \%$ de la población, un mejoramiento en las condiciones de vida, aunque algunas personas opinan que sólo será desde el punto de vista social y no económico.

$\mathrm{Al}$ incluir en el cuestionario que la población tendría una mayor participación en el proceso de desarrollo y conservación, hubo un 59\% de aceptación, ya que de esta forma no quedan excluidos y tendrán un espacio abierto a la concertación de opiniones, ya que la inclusión de la población al concepto de Parque Nacional es de vital importancia para su funcionamiento. Aunque hay un $12 \%$ de población que opinaron que también deberán acatar decisiones que no están acordes con sus intereses.

Respecto a las fuentes contaminantes un $48 \%$ de la población piensa en la necesidad de un mayor trabajo en la atenuación y control de las mismas tanto de origen estatal como particular y el $40 \%$ en un aumento del rigor de los inspectores en su control.

Como es lógico, con la instauración de un Area Protegida, es necesario para su protección poner en práctica regulaciones prohibitivas de utilización de la naturaleza, sólo un 39\% de la población está consciente de esto, por lo que es necesario educar a la comunidad en ese sentido para que no tomen ésto como una imposición que hay que cumplir, sino como algo que es imprescindible para la preservación de los ecosistemas naturales.

Un $61 \%$ de los habitantes de Viñales se sintieron beneficiados con el aumento de las fuentes de empleo que será necesario llevar a cabo para la instauración del Parque para su administración, y el estudio y protección de los valores naturales del lugar, ya que muchos mostraron la satisfacción que les daría trabajar o colaborar con el Parque Nacional para su buen funcionamiento.

Por todo lo antes expuesto y la necesidad de proteger la naturaleza, un $32 \%$ de la población opina y está de acuerdo que el Parque hará que desaparezcan los empleos clandestinos que utilizan las bellezas naturales de la región sin medida y para su beneficio, destruyendo los valores naturales del área con su comercialización y falta de conocimientos, lo que hace que la búsqueda de alternativas que solucionen este pro- 
blema en la Estrategia de Educación Ambiental sea de gran importancia.

Después de conocer la posición de las personas y qué piensan sobre el Parque Nacional Viñales se hace necesario saber sus conocimientos sobre los problemas ambientales que afectan el territorio. De forma general la población considera que los problemas ambientales de la región no son graves ni muy grandes, ya que los asocian con la industrialización que al ser escasa consideran que no hay.

A continuación daremos a conocer algunos de los problemas que afectan el Medio Ambiente en el área que ocupa el Parque Nacional Viñales, realizado por el gobierno municipal, los que serán confrontados después con los datos obtenidos en el cuestionario:

Problemas detectados en el territorio:

- Falta de organización, control y tratamiento de los desechos sólidos en el vertedero de Viñales.

- Problemas con la rehabilitación de los sistemas de tratamiento de residuales de los asentamientos, y centros productivos y económicos existentes.

- Contaminación del Río San Vicente con desechos sólidos, por falta de limpieza del mismo y la ausencia de depósitos para estos.
- Incumplimiento de las normas higiénico - sanitarias en el Asentamiento Humano Rural La Guasasa, en especial por la falta de abasto de agua.

- Realización de actividades ecoturísticas sin autorización.

A los problemas ambientales anteriores se suman otros, tales como la tala de bosques, señalada por un $46 \%$ de la población, con el fin de ampliar la frontera agrícola.

Al igual que existen personas preocupadas por la conservación, hay un $22 \%$ que no conocen los problemas ambientales que afectan el territorio viñalero y de éstos el $68,2 \%$ tiene un nivel de relación de medio a alto con el Parque Nacional, también encontramos que un $12 \%$ de la población de Viñales considera la inexistencia de problemas ambientales y de éstos el $41,6 \%$ es de un nivel de relación de medio a alto con e. Parque.

Otro de los problemas que un $17 \%$ de la población destaca es la caza de especies en veda o en vías de extinciór tanto para la alimentación como para lí comercialización o tan solo por entrete nimiento; otra preocupación es el verti miento de desechos en los ríos plantea do por un $14 \%$ de la población de Viñales, dada su gravedad. Los demá problemas ambientales que fueror planteados por la comunidad, aunqu 
no reúnen cada uno por su parte a más de un 10\% de población, no dejan de ser preocupantes. Como son, entre otros, la mala calidad de las aguas de consumo del Núcleo Urbano de Viñales, la utilización de pesticidas y fertilizantes químicos que contaminan los suelos y las aguas y la erosión de los suelos que puede traer graves problemas al municipio, ya que éste es eminentemente agrícola.

Como se observa, si bien existen coincidencias entre algunos de los problemas ambientales planteados anteriormente, no todos los habitantes de la comunidad conocen cuáles son los que afectan el área del Parque Nacional, de ahí que no se encuentren en condiciones para brindar soluciones, salvo algunos dirigentes, profesionales u otras personas que por su vinculación con estos tipos de problemas si están en condiciones de ofrecer alternativas.

A continuación se relacionan algunas de las soluciones propuestas por los habitantes entrevistados:

- Que funcionen los mecanismos de protección que están creados.

- La sustitución de pesticidas y fertilizantes químicos por productos naturales y biodegradables.

- La divulgación de los aspectos que agreden al Medio Ambiente para concientizar a la gente con la conservación.
- Diseñar y aplicar estrategias de Educación Ambiental desde las escuelas y toda la población.

- Regular la ampliación de la frontera agrícola.

- Declarar a Viñales como Parque Nacional.

Dada la necesidad de conocer, ante la declaración de Viñales como Area Protegida, la opinión de los habitantes de la comunidad sobre la relación que se deberá establecer entre la población y el Parque Nacional Vinales, fue incluida en el cuestionario una pregunta que recoge esta información.

Toda declaración de Parque Nacional trae transformaciones de las comunidades que dentro de ellas habitan, pero en Viñales existe un $31 \%$ de población que no tiene ni la más mínima idea de la relación que se debe establecer entre ambos. Por otra parte existe el mismo porcentaje que plantea que debe haber un vínculo de unión entre la comunidad y el Parque Nacional, donde se produzca un intercambio mutuo de conocimientos, por lo que un $18 \%$ de la población opinan que la administración del parque debe informar y educar a la comunidad, sin dejar de priorizar la educación ambiental en las escuelas, según un $2 \%$, para así incorporar la dimensión ambiental a niños, jóvenes y adultos. 
La contribución de la población es de vital importancia según un $25 \%$ de las opiniones concertadas, es decir, en el Parque Nacional Viñales debe haber una participación del pueblo para lograr que cada viñalero sienta suya esa relación hombre- naturaleza y que haga suyo ese concepto.

Muchas personas ven una posible afectación de esta relación con el desarrollo del turismo en la región, por lo que un $9 \%$ de ella planteó explícitamente que el Parque Nacional no sólo se dedique para el turismo, mientras que un $44 \%$ de ella lo reflejó implícitamente.

Un bajo porcentaje de población plantea que el Parque traerá ventajas y desventajas, aunque existe un $4 \%$ que piensa que sólo deben haber beneficios y no limitantes, lo que quiere decir que será necesario hacer un serio trabajo de concientización en la población para que sean capaces de asimilar las medidas de protección que serán implementadas. Si bien no deja de ser cierto que el Parque beneficiara en la medida de lo posible a las comunidades que dentro de él habitan, también tendrá que proteger la naturaleza del área y esto no sólo se logra con educación ambiental, también es necesario establecer restricciones de utilización de la naturaleza.

Para la buena elaboración de la Estrategia de Educación Ambiental fue necesario conocer cómo les sería más fácil a las personas recibir la información del funcionamiento de la región a partir de la declaración de Parque Nacional; como resultado se obtuvo que los medios de difusión masiva ocuparon la preferencia de los habitantes de Viñales y de ello los más acertados fueron la televisión y la radio ya que son los que llegan a todos los hogares, en tercer lugar se le dio gran importancia al trabajo de divulgación en centros de trabajo, escuelas y lugares públicos. La prensa escrita, por su frecuencia de llegada a los hogares ocupó el cuarto lugar en la preferencia de los viñaleros y las exposiciones en la galería según la opinión de la población podía jugar un importante papel en la divulgación y concientización de los habitantes del lugar.

El procesamiento de esta información nos permitió elaborar un Proyecto de Estrategia de Educación Ambiental ha seguir, el cual recoge "la expresión de la política ambiental de una región: en la cual se plasman sus proyecciones y directrices principales, con respecto a los procesos educativos a realizar en la comunidad» (elaborado por el autor) la cual sería un primer paso en la con certación de opiniones, para alcanzar tr participación comunitaria y el diálogc ambiental, para un futuro mejor mane jo, ya que la población local suele pose er amplios conocimientos de los recur sos existentes dentro de esas zonas! pueden ofrecer una importante contri 
bución a la conservación y junto a los gestores del Parque asegurar los medios en la búsqueda de fuentes de ingresos que reduzcan la presión sobre los recursos naturales.

Esta Estrategia de Educación Ambiental cuenta con un conjunto de acciones que permitirá lograr un dialogo ambiental donde nadie sea excluido y pueda expresarse- e intercambiar ideas, desarrollando así un rol más protagónico la comunidad en los procesos de desarrollo y conservación, aumentando su sentido de poder, mejorando su imagen, capacidad y autogestión, lo cual incide positivamente en la toma de decisiones, en el logro de proteger los recursos naturales, lo cual es de vital importancia en el mejoramiento de la calidad de vida y el bienestar de la población.

El conjunto de acciones que serán aplicadas a la comunidad para su educación y concientización ambiental debe seguir los lineamientos generales de la Estrategia Nacional de Educación Ambiental que son:

- Fortalecimiento de la capacidad institucional.

- Formación y capacitación de los recursos humanos.

- La dimensión ambiental en Educación Formal.

- La dimensión ambiental en los procesos de Educación no Formal.
- Desarrollo y fortalecimiento de la disponibilidad y acceso a la información.

- La dimensión ambiental en los procesos de comunicación y divulgación.

Las acciones que contiene esta estrategia persiguen de forma general dar a conocer todo lo referente a un Parque Nacional, instruir y educar a tomadores de decisiones, profesionales, lideres comunitarios y población en general en materia de medio ambiente, mitigar las fuentes contaminantes, enriquecer la enseñanza ambiental en las escuelas, eliminar la cacería furtiva e incorporar a la mujer al proceso de protección de la naturaleza debido a su posición en la familia y el trabajo. También se persigue mitigar las tendencias y efectos negativos que pueda traer la actividad turística en la región.

Al analizar los resultados del trabajo se obtuvo como principales conclusiones que dada la situación del país, la poca cultura ambiental y las necesidades que presenta la población asentada en el valle, hacen que se incremente la presión sobre los recursos florísticos y faunísticos que se pueden aprovechar, y que la población más difícil de vincular al proceso de protección y educación ambiental es la población sin vinculo laboral o académico. 
Se espera que a través de la futura aplicación del Proyecto de Estrategia de Educación Ambiental se logre concientizar a la población, para que la misma no sea hostil al Parque Nacional y así poderla incluir en este concepto para que el valle de Viñales por sus condiciones pase a formar parte del Parque Nacional Viñales con población incluida.
Esta relación parque - comunidad no podrá ser posible si la actividad turística no cambia sus actuales tendencias y pasa a ser compatible con la conservación de la naturaleza y de los valores patrimoniales, así como qué parte de las ganancias obtenidas en el territorio se inviertan en él, para mejorar las condiciones de vida de la población. 